\title{
Synthesis and biological evaluation of thieno[2,3-d]pyrimidine derivatives for anti-inflammatory, analgesic and ulcerogenic activity
}

\author{
ABDEL-RAHMAN B. A. EL-GAZZAR* \\ HODA A. R. HUSSEIN \\ HEND N. HAFEZ \\ Photochemistry Department \\ (Heterocyclic Unit) \\ National Research Center \\ Dokki, Giza, Egypt
}

Accepted September 19, 2007

\begin{abstract}
5-Methyl-6-phenyl-2-thioxothieno[2,3-d]pyrimidone derivative (2) reacted with hydrazonoyl chloride derivatives to afford triazolothienopyrimidones $\mathbf{4 a - f}$. Also, acetone-1-(2-amino-5-isopropyl-thiophene-3-carbonitrile) (3) reacted with functional and bifunctional groups to yield the corresponding compounds $\mathbf{5}-\mathbf{1 1}$. The new products showed anti-inflammatory, analgesic, and ulcerogenic activities comparable to that of indomethacin and acetylsalicylic acid, respectively.

Keywords: triazolothienopyrimidine, thienopyrimidines, thieno[2,3- $d][1,3]$ thiazine, thienopyridines, analgesic, anti-inflammatory, ulcerogenic activity
\end{abstract}

Pyrimidine and thienopyrimidine derivatives have attracted a great deal of interest owing to their medicinal activities (1-4). Pyrimidine derivatives and heterocyclic annelated pyrimidines continue to attract great interest due to the wide variety of interesting biological activities observed for these compounds, such as anticancer (4), antiviral (5), antitumor (6), anti-inflammatory (7) and antimicrobial activities (8). Also, the rapid growth in the literature dealing with the synthesis and biological activity of the thienopyrimidine derivatives prompted us to synthesize new derivatives of fused pyrimidine, thienopyrimidine and thienopyridine derivatives. In our previous work $(9,10)$, we reported the behaviour of thienopyrimidine derivatives towards hydrazines, 1,3-diketones, $\alpha$-haloketones and acids. As part of this work, here we report a new synthesis strategy for the preparation of functionalized thieno[2,3- $d][1,2,4]$ triazolo[ $4,5-a]$ pyrimidines, thieno $[2,3-d][1,3]$ thiazine and thienopyrimidine derivatives via the reaction of hydrazonyl chloride and cinnamonitriles with thienopyrimidone and 2-amino-5-isopropyl-thiophene-3-carbonitrile.

\footnotetext{
* Correspondence, e-mail: profelgazzar@yahoo.com
} 
A.-R. B. A. El-Gazzar et al.: Synthesis and biological evaluation of thieno[2,3-d]pyrimidine derivatives for anti-inflammatory, analgesic and ulcerogenic activity, Acta Pharm. 57 (2007) 395-411.

\section{EXPERIMENTAL}

All melting points were uncorrected and measured using an Electro-thermal IA 9100 apparatus (Shimadzu, Japan). Microanalytical data were performed with a Vario, Elementar apparatus (Shimadzu) (Table I). The IR spectra $(\mathrm{KBr})$ were recorded on a Perkin-Elmer 1650 spectrometer (USA). ${ }^{1} \mathrm{H}$ NMR spectra were determined on a Varian Mercury (300 MHz) spectrometer (Varian, UK) and chemical shifts were expressed in ppm relative to $\mathrm{SiMe}_{4}$ as internal standard. Mass spectra were recorded on 70 ev EI Ms-QP 1000 EX (Shimadzu) (Table II).

The starting materials 2-amino-4-methyl-5-phenyl-thiophene-3-ethylcarboxylate (1), 5-methyl-6-phenyl-2-thioxo-1,2,3,4-tetrahydro-thieno[2,3-d]pyrimidine-4-one (2) and acetone-1-(2-amino-5-isopropyl-thiophene-3-carbonitrile (3) were prepared according to Gewald et al. (11). The hydrazonoyl chloride was prepared as reported $(12,13)$.

Physico-chemical and spectral data of the synthesized compounds are given in Tables I and II, respectively.

\section{Synthesis of 2-amino-4-methyl-5-phenyl-thiopene-3-ethylcarboxylate (1)}

A mixture of 6-methyl-heptane-2,4-dione, ethylcyanoacetate, sulfur and diethylamine was heated $\left(70{ }^{\circ} \mathrm{C}\right)$ under stirring in absolute ethanol for 4 hours, then the mixture was left for 24 hours at $0{ }^{\circ} \mathrm{C}$. The formed solid was collected by filtration, washed with absolute ethanol $(20 \mathrm{~mL})$, dried and yellow crystals were obtained from absolute ethanol.

\section{Synthesis of 5-methyl-6-phenyl-2-thioxo-1,2,3,4-tetrahydro-thieno[2,3-d]pyrimidine- -4-one}

A mixture of compound $1(0.01 \mathrm{~mol})$ and potassium thiocyanate $(0.15 \mathrm{~mol})$ was stirred under reflux in dioxane containing $10 \% \mathrm{HCl}$ for 15 hours. Poured into $200 \mathrm{~mL}$ water, the solid precipitated was filtered off and crystallized from dioxane as yellow powder.

\section{Synthesis of acetone-1-(2-amino-5-isopropyl-thiophene-3-carbonitrile (3)}

A mixture of 6-methyl-heptane-2,4-dione, malononitrile, sulfur and diethylamine was heated $\left(70^{\circ} \mathrm{C}\right)$ under stirring in absolute ethanol for 4 hours, then left for 24 hours at $0{ }^{\circ} \mathrm{C}$. The formed solid was collected by filtration, washed with absolute ethanol $(20 \mathrm{~mL})$, dried and crystallized from absolute ethanol as yellow crystals.

Synthesis of thieno[2,3-d][1,2,4]triazolo[4,3-a]pyrimidin-5-one derivatives $(4 a-f)$. General procedure

A mixture of compound $2(2.74 \mathrm{~g}, 0.01 \mathrm{~mol})$ and the appropriate hydrazonoyl chloride $(0.01 \mathrm{~mol})$ was stirred under reflux in dry chloroform $(30 \mathrm{~mL})$ with 4 drops of triethylamine for 5 hours. The solvent was evaporated under reduced pressure. The 
A.-R. B. A. El-Gazzar et al.: Synthesis and biological evaluation of thieno[2,3-d]pyrimidine derivatives for anti-inflammatory, analgesic and ulcerogenic activity, Acta Pharm. 57 (2007) 395-411.

solid produced was washed three times with $30 \mathrm{~mL}$ absolute ethanol and crystallized from an appropriate solvent.

6-Methyl-1,3,7-triphenyl-thieno[2,3-d][1,2,4]triazolo[4,3-a]pyrimidin-5-one (4a). - Compound 4a was obtained from $2(2.74 \mathrm{~g}, 0.01 \mathrm{~mol})$ and $N$-phenylbenzene-carbohydrazonoyl chloride $(2.31 \mathrm{~g}, 0.01 \mathrm{~mol})$ as white needles, and crystallized from dimethylformamide.

3-Acetyl-1-(4-chlorophenyl)-7-phenyl-6-methyl-thieno[2,3-d][1,2,4]triazolo-[4,3-a]pyrimidin-5-one $(4 b)$. - Compound $4 \mathrm{~b}$ was obtained from $2(2.74 \mathrm{~g}, 0.01 \mathrm{~mol})$ and 2-oxo-N-(4-chlorophenyl)-propane hydrazonoyl chloride $(1.96 \mathrm{~g}, 0.01 \mathrm{~mol})$ as brown powder, and crystallized from dimethylformamide.

3-Acetyl-1-(4-methoxyphenyl)-7-phenyl-6-methyl-thieno[2,3-d][1,2,4]triazolo-[4,3-a]pyrimidin-5-one (4c). - Compound $4 \mathrm{c}$ was obtained from $2(2.74 \mathrm{~g}, 0.01 \mathrm{~mol})$ and 2-oxo-N-(4-methoxy-phenyl)-propane hydrazonoyl chloride $(1.91 \mathrm{~g}, 0.01 \mathrm{~mol})$ as green powder, and crystallized from absolute ethanol.

3-Acetyl-1-(4-nitrophenyl)-7-phenyl-6-methyl-thieno[2,3-d][1,2,4]triazolo[4,3-a] pyrimidin-5-one $(4 d)$. - Product $4 \mathrm{~d}$ was obtained from 2 (2.74 g, $0.01 \mathrm{~mol})$ and 2-oxo-N-(4-nitrophenyl)-propane hydrazonoyl chloride $(2.06 \mathrm{~g}, 0.01 \mathrm{~mol})$ as pale red crystals, and crystallized from absolute ethanol.

1,7-Diphenyl-6-methyl-thieno[2,3-d][1,2,4]triazolo[4,3-a]pyrimidin-5-one-3-ethylcarboxylate (4e). - Compound $4 \mathrm{e}$ was obtained from $2(2.74 \mathrm{~g}, 0.01 \mathrm{~mol})$ and chloro-(phenylhydrazono)-ethyl acetate $(2.27 \mathrm{~g}, 0.01 \mathrm{~mol})$ as white powder, and crystallized from dimethylformamide.

7-Phenyl-6-methyl-1-(4-tollyl)-thieno[2,3-d][1,2,4]triazolo[4,3-a]pyrimidin-5-one-3-ethylcarboxylate (4f). - Compound $4 \mathrm{f}$ was obtained from 2 (2.74 g, $0.01 \mathrm{~mol})$ and chloro-(4-tolylhydrazono)-ethyl acetate $(2.41 \mathrm{~g}, 0.01 \mathrm{~mol})$ as yellow powder, and crystallized from absolute ethanol/dioxane.

Synthesis of 6-isopropyl-5-(2-oxo-propyl)-3H-thieno[2,3-d]pyrimidin-4-one (5a)

A mixture of compound $3(2.22 \mathrm{~g}, 0.01 \mathrm{~mol})$, formic acid $(10 \mathrm{~mL})$ and catalytic amount of concentrated hydrochloric acid was heated under reflux for 12 hours. The reaction mixture was allowed to cool to room temperature, poured into water (100 mL). The formed solid was collected by filtration, washed with absolute ethanol $(20 \mathrm{~mL})$, dried and crystallized from dimethylformamide.

Synthesis of 6-isopropyl-2-methyl-5-(2-oxo-propyl)-3H-thieno[2,3-d]pyrimidin-4-one (5b)

A mixture of $3(2.22 \mathrm{~g}, 0.01 \mathrm{~mol})$ and glacial acetic acid $(30 \mathrm{~mL})$ was stirred under reflux for 12 hours (under TLC analysis). The reaction mixture was allowed to cool to room temperature and was poured into water $(100 \mathrm{~mL})$. The solid thus-formed was collected by filtration, washed with etanol $(20 \mathrm{~mL})$, dried and crystallized from dimethylformamide. 
A.-R. B. A. El-Gazzar et al.: Synthesis and biological evaluation of thieno[2,3-d]pyrimidine derivatives for anti-inflammatory, analgesic and ulcerogenic activity, Acta Pharm. 57 (2007) 395-411.

Synthesis of 1-(6-isopropyl-2,4-dithioxo-1,2,3,4-tetrahydro-thieno[2,3-d]pyrimidin-5-yl)-propan-2-one (6)

A mixture of compound 3 (2.22 g, $0.01 \mathrm{~mol}$ ) and carbon disulphide (excess $10 \mathrm{~mL}$ ) was heated under reflux in absolute ethanolic potassium hydroxide solution for 12 hours. The reaction mixture was allowed to cool to $0{ }^{\circ} \mathrm{C}$ for 3 hours, the deposited precipitate was filtered off, washed with water $(20 \mathrm{~mL})$, dried and crystallized from dioxane.

Synthesis of 1-(4-imino-6-isopropyl-2-thioxo-1,4-dihydro-2H-thieno[2,3-d][1,3]-thiazin-5-yl)-propan-2-one (7)

A mixture of compound $3(2.22 \mathrm{~g}, 0.01 \mathrm{~mol})$ and carbon disulphide (excess $10 \mathrm{~mL}$ ) was heated under reflux on a waterbath $\left(80^{\circ} \mathrm{C}\right.$ ) in $20 \mathrm{~mL}$ pyridine for 8 hours (under TLC control). The reaction mixture was allowed to cool to $0^{\circ} \mathrm{C}$ for 3 hours, the deposited precipitate was filtered off, washed with absolute ethanol $(20 \mathrm{~mL})$, dried and crystallized from dioxane.

Synthesis of 4-amino-6-isopropyl-5-(2-oxo-propyl)-1H-thieno[2,3-d]pyrimidin-(2-one or 2-thione) $(8 \boldsymbol{a}, \boldsymbol{b})$. General procedure

A mixture of compound $3(2.22 \mathrm{~g}, 0.01 \mathrm{~mol})$ and urea or thiourea $(0.01 \mathrm{~mol})$ was heated at $180^{\circ} \mathrm{C}$ in a test tube on sand-bath for 4 hours. The mixture was allowed to cool to room temperature; the product was solidified by cooling and addition of absolute ethanol $(50 \mathrm{~mL})$. The precipitate thus-formed was collected by filtration and crystallized from an appropriate solvent.

4-Amino-6-isopropyl-5-(2-oxo-propyl)-1H-thieno[2,3-d]pyrimidin-2-one (8a). - Compound $8 \mathbf{a}$ was obtained from 3 and urea $(0.61 \mathrm{~g}, 0.01 \mathrm{~mol})$, as dark brown crystals and crystallized from dimethylformamide.

4-Amino-6-isopropyl-5-(2-oxo-propyl)-1H-thieno[2,3-d]pyrimidine-2-thione (8b). - Compound $8 \mathbf{b}$ was obtained from $\mathbf{3}$ and thiourea $(0.77 \mathrm{~g}, 0.01 \mathrm{~mol})$ as brown crystals and crystallized from dioxane.

Synthesis of 4,6-diamino-2-isopropyl-3-(2-oxo-propyl)-thieno[2,3-b]pyridine-5-carbonitrile (9)

To a solution of $3(2.22 \mathrm{~g}, 0.01 \mathrm{~mol})$ in glacial acetic acid $(50 \mathrm{~mL})$, malononitrile $(0.66 \mathrm{~g}$, $0.01 \mathrm{~mol}$ ) was added. The reaction mixture was stirred under reflux for 8 hours, cooled to room temperature, then poured into cold water $(100 \mathrm{~mL})$. The solid product was filtered off, washed with water, dried and crystallized from absolute ethanol.

Synthesis of 4-amino-6-hydroxy-2-isopropyl-3-(2-oxo-propyl)-thieno[2,3-b]pyridine-5-carbonitrile (10)

A mixture of $3(2.22 \mathrm{~g}, 0.01 \mathrm{~mol})$ and ethylcyanoacetate $(1.13 \mathrm{~g}, 0.01 \mathrm{~mol})$ in dry dixane $(50 \mathrm{~mL})$ containing a catalytic amount of triethylamine was stirred under reflux for 8 hours. The reaction mixture was allowed to cool and poured into cold water (100 
A.-R. B. A. El-Gazzar et al.: Synthesis and biological evaluation of thieno[2,3-d]pyrimidine derivatives for anti-inflammatory, analgesic and ulcerogenic activity, Acta Pharm. 57 (2007) 395-411.

$\mathrm{mL}$ ) and neutralized with acetic acid. The precipitate was filtered off, washed with water, dried and crystallized from dioxane.

Synthesis of N-[3-cyano-5-isopropyl-4-(2-oxo-propyl)-thiophen-2-yl]-formamidic acid ethyl ester (11a)

A mixture of compound 3 (2.22 g, $0.01 \mathrm{~mol})$ and triethylorthoformate $(2.96 \mathrm{~g}, 20 \mathrm{mmol})$ was stirred under reflux in acetic anhydride $(30 \mathrm{~mL})$ for 6 hours. The reaction mixture was allowed to cool to room temperature, poured into cold water $(100 \mathrm{~mL})$ and neutralized by ammonia solution. The deposited precipitate was collected by filtration, washed with water, dried and crystallized from absolute ethanol.

\section{Synthesis of N-[3-cyano-5-isopropyl-4-(2-oxo-propyl)-thiophen-2-yl]-acetimidic acid} ethyl ester (11b)

A mixture of compound $3(2.22 \mathrm{~g}, 0.01 \mathrm{~mol})$ and triethylorthoacetate $(2.96 \mathrm{~g}, 20 \mathrm{mmol})$ was stirred under reflux in acetic anhydride $(30 \mathrm{~mL})$ for 6 hours. The reaction mixture was allowed to cool to room temperature, poured into cold water $(100 \mathrm{~mL})$ and neutralized by ammonia solution. The deposited precipitate was collected by filtration, washed with water, dried and crystallized from benzene.

Synthesis of N-[3-cyano-5-isopropyl-4-(2-oxo-propyl)-thiophen-2-yl]-formamidine (12)

A mixture of 11a $(2.78 \mathrm{~g}, 0.01 \mathrm{~mol})$ and ammonia solution $(35 \%, 20 \mathrm{~mL})$ was stirred under reflux in absolute ethanol $(20 \mathrm{~mL})$ for 8 hours. The reaction mixture was allowed to cool to room temperature, poured into cold water $(100 \mathrm{~mL})$ and neutrallized by diluted hydrochloric acid. The deposited white powder precipitate was collected by filtration, washed with water, dried and crystallized from absolute ethanol.

\section{Synthesis of 1-(4-amino-6-isopropyl-thieno[2,3-d]pyrimidin-5-yl)-propan-2-one (13)}

To a warmed absolute ethanolic sodium ethoxide solution [prepared by dissolving $0.23 \mathrm{~g}(0.01 \mathrm{~mol})$ sodium metal in $50 \mathrm{~mL}$ absolute ethanol], compound 12 (2.49 g, 0.01 mol) was added. The mixture was stirred under reflux for 8 hours. The reaction mixture was allowed to cool to room temperature, poured into cold water $(100 \mathrm{~mL})$ and neutralized by acetic acid. The deposited precipitate was filtered off, dried and crystallized from benzene.

Synthesis of 1-(3-amino-4-imino-6-isopropyl-3,4-dihydro-thieno[2,3-d]pyrimidin-5-yl)-propan-2-one (14)

A mixture of 11a $(2.78 \mathrm{~g}, 0.01 \mathrm{~mol})$ and hydrazine hydrate $(99-100 \%)(10 \mathrm{~mL})$ was stirred in absolute ethanol $(20 \mathrm{~mL})$ for 2 hours. The reaction mixture was allowed to cool to room temperature, poured into cold water $(100 \mathrm{~mL})$ and neutralized by acetic acid. The deposited precipitate was collected by filtration, washed with water, dried and crystallized from dioxane. 
PHARMACOLOGICAL SCREENING

\section{Animals}

Both sexes of Swiss albino mice (25-30 g) were used in analgesic activity testing and adult female Sprague-Dawley rats (150-180 g) were used in anti-inflammatory and ulcerogenic testing. International principle and local regulations concerning the care of used laboratory animals was taken into account (14). The animals had free access to standard commercial diet and water ad libitum and were kept in rooms maintained at $22 \pm 1$ ${ }^{\circ} \mathrm{C}$ with a $12 \mathrm{~h}$ light/dark cycle.

\section{Anti-inflammatory activity}

The method adopted resembles essentially that described by Winter et al. (15). Tween$-80(10 \%, V / V)$ was selected as vehicle to suspend the standard drug and test compounds. The rats were starved for $18 \mathrm{~h}$ prior to the experiment. The animals were weighed, marked for identification and divided into 14 groups, each containing 6 animals. Edema was induced in the left hind paw of all rats by subcutaneous injection of $0.1 \mathrm{~mL}$ of $1 \%$ $(\mathrm{m} / \mathrm{V})$ aqueous carrageenean into their footpads. The $1^{\text {st }}$ group was kept as control and was given $1.0 \mathrm{~mL}$ of the vehicle. The $2^{\text {nd }}$ to $13^{\text {th }}$ groups were orally administered an aqueous suspension of the synthesized compounds ( $15 \mathrm{mg} \mathrm{kg}^{-1}$ body mass) 1 hour before carrageenean injection. The last group (standard) was administered indomethacin in a dose of $10 \mathrm{mg} \mathrm{kg}^{-1}$ body mass, orally as suspension in $10 \%$ Tween 80 (16). The paw volume of each rat was measured with a mercury plethysmometer, before carrageenean injection and then hourly for 4 hours post administration of the suspension of synthesized compound in $10 \%$ Tween 80 .

\section{Analgesic activity}

The compounds $\mathbf{4 a - f}, \mathbf{5 a}, \mathbf{b}, \mathbf{6}, \mathbf{7}$ and $\mathbf{8} \mathbf{a}, \mathbf{b}$ were selected for investigating their analgesic activity in acetic acid induced writhing response in mice, following the method of Collier et al. (17). Eighty four mice were divided into 14 groups (six in each) and starved for $16 \mathrm{~h}$. The $1^{\text {st }}$ group which served as control was orally given distilled water in an appropriate volume. The $2^{\text {nd }}$ to $13^{\text {th }}$ groups received the aqueous suspension of synthesized compounds orally ( $15 \mathrm{mg} \mathrm{kg}^{-1}$ body mass). The last group received acetylsalicylic acid orally in a dose of $100 \mathrm{mg} \mathrm{kg}^{-1}$ body mass. After 30 minutes, each mouse was administerated $0.7 \%$ of an aqueous solution of acetic acid $\left(10 \mathrm{~mL} \mathrm{~kg}^{-1}\right.$ body mass) and the mice were then placed in transparent boxes for observation. The number of writhes was counted for 20 min after acetic acid injection. The number of writhes in each treated group was compared to that of the control group. The number of writhings was recorded and the percentage protection was calculated.

\section{Ulcerogenicity}

Ulceration in rats was induced as described by Goel et al. (18). Rats were divided into 14 groups, of six animals each. The control group of animals was administered only 
A.-R. B. A. El-Gazzar et al.: Synthesis and biological evaluation of thieno[2,3-d]pyrimidine derivatives for anti-inflammatory, analgesic and ulcerogenic activity, Acta Pharm. 57 (2007) 395-411.

Table I. Physical and chemical properties of synthesized compounds

\begin{tabular}{|c|c|c|c|c|c|c|}
\hline \multirow{2}{*}{$\begin{array}{l}\text { Compd. } \\
\text { No. }\end{array}$} & \multirow{2}{*}{$\begin{array}{l}\text { Yield } \\
(\%)\end{array}$} & \multirow{2}{*}{$\begin{array}{l}\text { M.p. } \\
\left({ }^{\circ} \mathrm{C}\right)\end{array}$} & \multirow{2}{*}{$\begin{array}{l}\text { Mol. formula } \\
\quad\left(M_{\mathrm{r}}\right)\end{array}$} & \multicolumn{3}{|c|}{ Found/calcd. (\%) } \\
\hline & & & & C & $\mathrm{H}$ & $\mathrm{N}$ \\
\hline 1 & 86 & $94-96$ & $\begin{array}{c}\mathrm{C}_{14} \mathrm{H}_{15} \mathrm{NO}_{2} \mathrm{~S} \\
(262.33)\end{array}$ & $\begin{array}{l}64,35 \\
64.32\end{array}$ & $\begin{array}{l}5.78 \\
5.75\end{array}$ & $\begin{array}{l}5.36 \\
5.38\end{array}$ \\
\hline 2 & 81 & $313-316$ & $\begin{array}{c}\mathrm{C}_{13} \mathrm{H}_{10} \mathrm{~N}_{2} \mathrm{OS}_{2} \\
(274.35)\end{array}$ & $\begin{array}{l}56.90 \\
56.80\end{array}$ & $\begin{array}{l}3.67 \\
4.00\end{array}$ & $\begin{array}{l}10.21 \\
10.40\end{array}$ \\
\hline 3 & 73 & $267-270$ & $\begin{array}{c}\mathrm{C}_{11} \mathrm{H}_{14} \mathrm{~N}_{2} \mathrm{OS} \\
(222.31)\end{array}$ & $\begin{array}{l}59.43 \\
59.38\end{array}$ & $\begin{array}{l}6.35 \\
6.32\end{array}$ & $\begin{array}{l}12.60 \\
12.56\end{array}$ \\
\hline $4 a$ & 66 & $239-241$ & $\begin{array}{c}\mathrm{C}_{26} \mathrm{H}_{18} \mathrm{~N}_{4} \mathrm{OS} \\
(434.52)\end{array}$ & $\begin{array}{l}71.87 \\
71.84\end{array}$ & $\begin{array}{l}4.17 \\
4.15\end{array}$ & $\begin{array}{l}12.89 \\
12.76\end{array}$ \\
\hline $4 b$ & 70 & $301-303$ & $\begin{array}{c}\mathrm{C}_{22} \mathrm{H}_{15} \mathrm{ClN}_{4} \mathrm{O}_{2} \mathrm{~S} \\
(434.90)\end{array}$ & $\begin{array}{l}60.76 \\
60.64\end{array}$ & $\begin{array}{l}3.48 \\
3.50\end{array}$ & $\begin{array}{l}12.88 \\
12.79\end{array}$ \\
\hline $4 c$ & 68 & $301-303$ & $\begin{array}{c}\mathrm{C}_{23} \mathrm{H}_{18} \mathrm{~N}_{4} \mathrm{O}_{3} \mathrm{~S} \\
(430.48)\end{array}$ & $\begin{array}{l}64.17 \\
64.11\end{array}$ & $\begin{array}{l}4.21 \\
4.18\end{array}$ & $\begin{array}{l}13.01 \\
12.89\end{array}$ \\
\hline $4 d$ & 69 & $287-290$ & $\begin{array}{c}\mathrm{C}_{22} \mathrm{H}_{15} \mathrm{~N}_{5} \mathrm{O}_{4} \mathrm{~S} \\
(445.46)\end{array}$ & $\begin{array}{l}59.32 \\
59.29\end{array}$ & $\begin{array}{l}3.39 \\
3.30\end{array}$ & $\begin{array}{l}15.72 \\
15.67\end{array}$ \\
\hline $4 e$ & 72 & $276-278$ & $\begin{array}{c}\mathrm{C}_{23} \mathrm{H}_{18} \mathrm{~N}_{4} \mathrm{O}_{3} \mathrm{~S} \\
(430.49)\end{array}$ & $\begin{array}{l}64.17 \\
64.13\end{array}$ & $\begin{array}{l}4.21 \\
4.19\end{array}$ & $\begin{array}{l}13.01 \\
12.89\end{array}$ \\
\hline $4 \mathrm{f}$ & 70 & $243-245$ & $\begin{array}{c}\mathrm{C}_{24} \mathrm{H}_{20} \mathrm{~N}_{4} \mathrm{O}_{3} \mathrm{~S} \\
(444.51)\end{array}$ & $\begin{array}{l}64.85 \\
64.76\end{array}$ & $\begin{array}{l}4.53 \\
4.48\end{array}$ & $\begin{array}{l}12.60 \\
12.54\end{array}$ \\
\hline $5 a$ & 76 & $278-281$ & $\begin{array}{c}\mathrm{C}_{12} \mathrm{H}_{14} \mathrm{~N}_{2} \mathrm{O}_{2} \mathrm{~S} \\
(250.32)\end{array}$ & $\begin{array}{l}57.58 \\
57.49\end{array}$ & $\begin{array}{l}5.64 \\
5.61\end{array}$ & $\begin{array}{l}11.19 \\
11.08\end{array}$ \\
\hline $5 b$ & 78 & $256-258$ & $\begin{array}{c}\mathrm{C}_{13} \mathrm{H}_{16} \mathrm{~N}_{2} \mathrm{O}_{2} \mathrm{~S} \\
(264.35)\end{array}$ & $\begin{array}{l}59.07 \\
59.02\end{array}$ & $\begin{array}{l}6.10 \\
6.03\end{array}$ & $\begin{array}{l}10.60 \\
10.54\end{array}$ \\
\hline 6 & 65 & $321-324$ & $\begin{array}{c}\mathrm{C}_{12} \mathrm{H}_{14} \mathrm{~N}_{2} \mathrm{OS}_{3} \\
(298.45)\end{array}$ & $\begin{array}{l}48.29 \\
48.22\end{array}$ & $\begin{array}{l}4.73 \\
4.67\end{array}$ & $\begin{array}{l}9.39 \\
9.28\end{array}$ \\
\hline 7 & 59 & $289-292$ & $\begin{array}{c}\mathrm{C}_{12} \mathrm{H}_{14} \mathrm{~N}_{2} \mathrm{OS}_{3} \\
(298.45)\end{array}$ & $\begin{array}{l}48.29 \\
48.26\end{array}$ & $\begin{array}{l}4.73 \\
4.70\end{array}$ & $\begin{array}{l}9.39 \\
9.35\end{array}$ \\
\hline $8 a$ & 68 & $341-343$ & $\begin{array}{c}\mathrm{C}_{12} \mathrm{H}_{15} \mathrm{~N}_{3} \mathrm{O}_{2} \mathrm{~S} \\
(265.33)\end{array}$ & $\begin{array}{l}54.32 \\
54.29\end{array}$ & $\begin{array}{l}5.69 \\
5.71\end{array}$ & $\begin{array}{l}15.83 \\
15.79\end{array}$ \\
\hline $8 b$ & 64 & $316-319$ & $\begin{array}{c}\mathrm{C}_{12} \mathrm{H}_{15} \mathrm{~N}_{3} \mathrm{OS}_{2} \\
(281.40)\end{array}$ & $\begin{array}{l}51.21 \\
51.18\end{array}$ & $\begin{array}{l}5.37 \\
5.38\end{array}$ & $\begin{array}{l}14.93 \\
14.87\end{array}$ \\
\hline 9 & 63 & $262-264$ & $\begin{array}{c}\mathrm{C}_{14} \mathrm{H}_{16} \mathrm{~N}_{4} \mathrm{OS} \\
(288.37)\end{array}$ & $\begin{array}{l}58.31 \\
58.27\end{array}$ & $\begin{array}{l}5.59 \\
5.54\end{array}$ & $\begin{array}{l}19.42 \\
19.36\end{array}$ \\
\hline 10 & 61 & $278-280$ & $\begin{array}{c}\mathrm{C}_{14} \mathrm{H}_{15} \mathrm{~N}_{3} \mathrm{O}_{2} \mathrm{~S} \\
(289.36)\end{array}$ & $\begin{array}{l}58.11 \\
58.08\end{array}$ & $\begin{array}{l}5.22 \\
5.17\end{array}$ & $\begin{array}{l}14.52 \\
14.49\end{array}$ \\
\hline $11 a$ & 74 & 195-197 & $\begin{array}{c}\mathrm{C}_{14} \mathrm{H}_{18} \mathrm{~N}_{2} \mathrm{O}_{2} \mathrm{~S} \\
(278.37)\end{array}$ & $\begin{array}{l}60.40 \\
60.46\end{array}$ & $\begin{array}{l}6.51 \\
6.50\end{array}$ & $\begin{array}{l}10.06 \\
10.11\end{array}$ \\
\hline $11 b$ & 71 & $207-209$ & $\begin{array}{c}\mathrm{C}_{15} \mathrm{H}_{20} \mathrm{~N}_{2} \mathrm{O}_{2} \mathrm{~S} \\
(292.40)\end{array}$ & $\begin{array}{l}61.61 \\
61.57\end{array}$ & $\begin{array}{l}6.89 \\
6,84\end{array}$ & $\begin{array}{l}9.58 \\
9.61\end{array}$ \\
\hline 12 & 56 & $261-263$ & $\begin{array}{c}\mathrm{C}_{12} \mathrm{H}_{15} \mathrm{~N}_{3} \mathrm{OS} \\
(249.33)\end{array}$ & $\begin{array}{l}57.80 \\
57.77\end{array}$ & $\begin{array}{l}6.06 \\
6.00\end{array}$ & $\begin{array}{l}16.85 \\
16.76\end{array}$ \\
\hline 13 & 66 & $309-311$ & $\begin{array}{c}\mathrm{C}_{12} \mathrm{H}_{15} \mathrm{~N}_{3} \mathrm{OS} \\
(249.33)\end{array}$ & $\begin{array}{l}57.81 \\
57.76\end{array}$ & $\begin{array}{l}6.06 \\
6.02\end{array}$ & $\begin{array}{l}16.85 \\
16.91\end{array}$ \\
\hline 14 & 68 & $259-162$ & $\begin{array}{c}\mathrm{C}_{12} \mathrm{H}_{16} \mathrm{~N}_{4} \mathrm{OS} \\
(264.35)\end{array}$ & $\begin{array}{l}54.52 \\
54.49\end{array}$ & $\begin{array}{l}6.10 \\
6.09\end{array}$ & $\begin{array}{l}21.19 \\
21.07\end{array}$ \\
\hline
\end{tabular}


A.-R. B. A. El-Gazzar et al.: Synthesis and biological evaluation of thieno[2,3-d]pyrimidine derivatives for anti-inflammatory, analgesic and ulcerogenic activity, Acta Pharm. 57 (2007) 395-411.

Table II. Spectral data of the synthesized compounds

\begin{tabular}{|c|c|c|c|}
\hline $\begin{array}{l}\text { Compd. } \\
\text { No. }\end{array}$ & $\begin{array}{l}\text { Mass } \\
(\mathrm{m} / \mathrm{z})\end{array}$ & $\begin{array}{c}\mathrm{IR} \\
\left(v, \mathrm{~cm}^{-1}\right)\end{array}$ & $\begin{array}{l}{ }^{1} \mathrm{H} \text { NMR }(\delta, \mathrm{ppm}) \\
\left(\mathrm{DMSO}-d_{6}\right)\end{array}$ \\
\hline 1 & $\begin{array}{l}261\left(\mathrm{M}^{+}\right)(100) \\
188\left(\mathrm{M}^{+}-73\right)(31)\end{array}$ & $45\left(\mathrm{NH}_{2}\right)$ & $\begin{array}{l}1.21\left(\mathrm{t}, 3 \mathrm{H}, \mathrm{CH}_{3}\right), 2.38\left(\mathrm{~s}, 3 \mathrm{H}, \mathrm{CH}_{3}\right), 4.01(\mathrm{q}, 2 \mathrm{H}, \\
\left.\mathrm{CH}_{2}\right), 7.43-7.52(\mathrm{~m}, 5 \mathrm{H}, \text { phenyl), } 8.23 \text { (brs, } 2 \mathrm{H}, \\
\mathrm{NH}_{2}, \mathrm{D}_{2} \mathrm{O} \text { exchangeable) }\end{array}$ \\
\hline 2 & $274\left(\mathrm{M}^{+}\right),(100)$ & $\begin{array}{l}3153(\mathrm{NH}) \\
1675(\mathrm{C}=\mathrm{O})\end{array}$ & $\begin{array}{l}2,40\left(\mathrm{~s}, 3 \mathrm{H}, \mathrm{CH}_{3}\right), 7.51(\mathrm{~m}, 5 \mathrm{H}, \text { phenyl), } 12.45(\mathrm{~s}, \\
\left.1 \mathrm{H}, \mathrm{NH}, \mathrm{D}_{2} \mathrm{O} \text { exchangeable }\right), 13.50(\mathrm{~s}, 1 \mathrm{H}, \mathrm{NH}, \\
\mathrm{D}_{2} \mathrm{O} \text { exchangeable) }\end{array}$ \\
\hline 3 & $222\left(\mathrm{M}^{+}\right)(100)$ & $\begin{array}{l}3400\left(\mathrm{brs}, \mathrm{NH}_{2}\right) \\
1704(\mathrm{C}=\mathrm{O}) \\
2217(\mathrm{CN})\end{array}$ & $\begin{array}{l}1.00\left(\mathrm{~d}, 6 \mathrm{H}, 2 \mathrm{CH}_{3}\right), 2.05(\text { Septet, } 1 \mathrm{H}, \mathrm{CH}), 2.40(\mathrm{~s}, \\
\left.3 \mathrm{H}, \mathrm{CH}_{3}\right), 2.50\left(\mathrm{~s}, 2 \mathrm{H}, \mathrm{CH}_{2}\right), 13.30(\mathrm{brs}, 1 \mathrm{H}, \mathrm{NH}, \\
\mathrm{D}_{2} \mathrm{O} \text { exchangable) }\end{array}$ \\
\hline $4 a$ & $\begin{array}{l}434\left(\mathrm{M}^{+}\right)(100) \\
357\left(\mathrm{M}^{+}-77\right)(28)\end{array}$ & $1686(\mathrm{C}=\mathrm{O})$ & $\begin{array}{l}2.11\left(\mathrm{~s}, 3 \mathrm{H}, \mathrm{CH}_{3}\right), 7.20-7.30(\mathrm{~m}, 6 \mathrm{H}, \text { phenyl), } 7.40 \\
7.56(\mathrm{~m}, 9 \mathrm{H}, \text { phenyl protons })\end{array}$ \\
\hline $4 b$ & $\begin{array}{l}434(100) \\
391\left(\mathrm{M}^{+}-43\right)(54)\end{array}$ & $\begin{array}{l}1700,1686 \\
(2 \mathrm{C}=\mathrm{O})\end{array}$ & $\begin{array}{l}2.13\left(\mathrm{~s}, 3 \mathrm{H}, \mathrm{CH}_{3}\right), 2.62\left(\mathrm{~s}, 3 \mathrm{H}, \mathrm{CH}_{3}\right), 7.10-7.15(\mathrm{~d}, \\
2 \mathrm{H}, \text { phenyl), } 7.30-7.35(\mathrm{~m}, 2 \mathrm{H}, \text { phenyl), } 7.42-7.44 \\
(\mathrm{m}, 3 \mathrm{H}, \text { phenyl }), 7.65-7.68(\mathrm{~d}, 2 \mathrm{H}, \text { phenyl })\end{array}$ \\
\hline $4 c$ & $\begin{array}{l}430(100) \\
399\left(\mathrm{M}^{+}-31\right)(32) \\
387\left(\mathrm{M}^{+}-43\right)(25)\end{array}$ & $\begin{array}{l}1702,1687 \\
(2 \mathrm{C}=\mathrm{O})\end{array}$ & $\begin{array}{l}2.11\left(\mathrm{~s}, 3 \mathrm{H}, \mathrm{CH}_{3}\right), 2.64\left(\mathrm{~s}, 3 \mathrm{H}, \mathrm{CH}_{3}\right), 3.88(\mathrm{~s}, 3 \mathrm{H}, \\
\left.\mathrm{OCH}_{3}\right), 7.15-7.20(\mathrm{~d}, 2 \mathrm{H}, \mathrm{p}-\mathrm{sub}-\mathrm{pheny}), 7.24-7.30 \\
(\mathrm{~m}, 3 \mathrm{H}, \text { phenyl), } 7.47-7.56(\mathrm{~m}, 2 \mathrm{H} \text {, phenyl), 7.59- } \\
7.63(\mathrm{~d}, 2 \mathrm{H} \text {, phenyl) }\end{array}$ \\
\hline $4 d$ & $\begin{array}{l}456\left(\mathrm{M}^{+}+1\right)(34) \\
445(100) \\
402\left(\mathrm{M}^{+}-43\right)(22)\end{array}$ & $\begin{array}{l}1705,1683 \\
(2 \mathrm{C}=\mathrm{O})\end{array}$ & $\begin{array}{l}2.14\left(\mathrm{~s}, 3 \mathrm{H}, \mathrm{CH}_{3}\right), 2.80\left(\mathrm{~s}, 3 \mathrm{H}, \mathrm{CH}_{3}\right), 7.16-7.22(\mathrm{~d}, \\
2 \mathrm{H}, \text { pheny), } 7.26-7.35(\mathrm{~m}, 3 \mathrm{H}, \text { phenyl), } 7.51-7.57 \\
(\mathrm{m}, 2 \mathrm{H}, \text { phenyl }), 7.56-7.60(\mathrm{~d}, 2 \mathrm{H}, \text { phenyl })\end{array}$ \\
\hline $4 e$ & $430\left(\mathrm{M}^{+}\right)(100)$ & $\begin{array}{l}1714,1689 \\
(2 \mathrm{C}=\mathrm{O})\end{array}$ & $\begin{array}{l}1.27\left(\mathrm{t}, 3 \mathrm{H}, \mathrm{CH}_{3}\right), 2.13\left(\mathrm{~s}, 3 \mathrm{H}, \mathrm{CH}_{3}\right), 4.35(\mathrm{q}, 2 \mathrm{H}, \\
\left.\mathrm{CH}_{2}\right), 7.25-7.30(\mathrm{~m}, 6 \mathrm{H} \text {, pheny), } 7.40-7.75(\mathrm{~m}, 4 \mathrm{H}, \\
\text { phenyl) }\end{array}$ \\
\hline $4 \mathrm{f}$ & $\begin{array}{l}444\left(\mathrm{M}^{+}\right)(100) \\
415\left(\mathrm{M}^{+}-29\right) \\
((12)\end{array}$ & $\begin{array}{l}1715,1689 \\
(2 \mathrm{C}=\mathrm{O})\end{array}$ & $\begin{array}{l}1.28\left(\mathrm{t}, 3 \mathrm{H}, \mathrm{CH}_{3}\right), 2.10\left(\mathrm{~s}, 3 \mathrm{H}, \mathrm{CH}_{3}\right), 2.35(\mathrm{~s}, 3 \mathrm{H}, \\
\left.\mathrm{CH}_{3}\right), 4.38\left(\mathrm{q}, 2 \mathrm{H}, \mathrm{CH}_{2}\right), 7.12-7.16(\mathrm{~d}, 2 \mathrm{H}, \mathrm{phenyl}) \text {, } \\
7.25-7.35(\mathrm{~m}, 3 \mathrm{H}, \mathrm{pheny}), 7.40-7.51(\mathrm{~m}, 2 \mathrm{H}, \text { phe- } \\
\text { nyl), 8.00-8.05 (d, 2H, phenyl) }\end{array}$ \\
\hline $5 a$ & $250\left(\mathrm{M}^{+}\right)(100)$ & $\begin{array}{l}3355(\mathrm{brs}, \mathrm{NH}) \\
1705,1688 \\
(2 \mathrm{C}=\mathrm{O})\end{array}$ & $\begin{array}{l}1.02\left(\mathrm{~d}, 6 \mathrm{H}, 2 \mathrm{CH}_{3}\right), 2.07(\text { Septet, } 1 \mathrm{H}, \mathrm{CH}), 2.41(\mathrm{~s}, \\
\left.3 \mathrm{H}, \mathrm{CH}_{3}\right), 2.52\left(\mathrm{~s}, 2 \mathrm{H}, \mathrm{CH}_{2}\right), 8.21(\mathrm{~s}, 1 \mathrm{H}, \text { pyrimi- } \\
\text { dine proton), } 13.10 \text { (brs, } 1 \mathrm{H}, \mathrm{NH}, \mathrm{D}_{2} \mathrm{O} \text { exchan- } \\
\text { gable) }\end{array}$ \\
\hline $5 b$ & $264\left(\mathrm{M}^{+}\right)(100)$ & $\begin{array}{l}3316(\text { brs, NH) } \\
1700,1678 \\
(2 \mathrm{C}=\mathrm{O})\end{array}$ & $\begin{array}{l}1.01\left(\mathrm{~d}, 6 \mathrm{H}, 2 \mathrm{CH}_{3}\right), 2.06(\text { Septet, } 1 \mathrm{H}, \mathrm{CH}), 2,23(\mathrm{~s}, \\
\left.3 \mathrm{H}, \mathrm{CH}_{3}\right), 2.42\left(\mathrm{~s}, 3 \mathrm{H}, \mathrm{CH}_{3}\right), 2.48\left(\mathrm{~s}, 2 \mathrm{H}, \mathrm{CH}_{2}\right), 8.21 \\
(\mathrm{~s}, 1 \mathrm{H}, \text { pyrimidine proton), } 13.10(\mathrm{brs}, 1 \mathrm{H}, \mathrm{NH})\end{array}$ \\
\hline 6 & & $\begin{array}{l}3390(\text { brs, NH) } \\
1702(\mathrm{C}=\mathrm{O}) \\
1350,1365 \\
(2 \mathrm{C}=\mathrm{S})\end{array}$ & $\begin{array}{l}1.00\left(\mathrm{~d}, 6 \mathrm{H}, 2 \mathrm{CH}_{3}\right), 2.05(\text { Septet, } 1 \mathrm{H}, \mathrm{CH}), 2.40(\mathrm{~s}, \\
\left.3 \mathrm{H}, \mathrm{CH}_{3}\right), 2.50\left(\mathrm{~s}, 2 \mathrm{H}, \mathrm{CH}_{2}\right), 8.90(\mathrm{brs}, 1 \mathrm{H}, \mathrm{NH}, \\
\mathrm{D}_{2} \mathrm{O} \text { exchangeable), } 13.10(\text { brs, } 1 \mathrm{H}, \mathrm{NH})\end{array}$ \\
\hline 7 & $\begin{array}{l}298\left(\mathrm{M}^{+}\right)(66) \\
255\left(\mathrm{M}^{+}-43\right)(32) \\
241\left(\mathrm{M}^{+}-57\right)(42)\end{array}$ & $\begin{array}{l}3400(\text { brs, NH) } \\
1706(\mathrm{CO}) \\
1380(\mathrm{C}=\mathrm{S})\end{array}$ & $\begin{array}{l}1.00\left(\mathrm{~d}, 6 \mathrm{H}, 2 \mathrm{CH}_{3}\right), 2.04(\text { Septet, } 1 \mathrm{H}, \mathrm{CH}), 2.38(\mathrm{~s}, \\
\left.3 \mathrm{H}, \mathrm{CH}_{3}\right), 2.50\left(\mathrm{~s}, 2 \mathrm{H}, \mathrm{CH}_{2}\right), 8.02(\text { brs, } 1 \mathrm{H}, \mathrm{NH}) \text {, } \\
13.00(\text { brs }, 1 \mathrm{H}, \mathrm{NH})\end{array}$ \\
\hline
\end{tabular}


A.-R. B. A. El-Gazzar et al.: Synthesis and biological evaluation of thieno[2,3-d]pyrimidine derivatives for anti-inflammatory, analgesic and ulcerogenic activity, Acta Pharm. 57 (2007) 395-411.

Table II. continued

\begin{tabular}{|c|c|c|c|}
\hline $\begin{array}{l}\text { Compd. } \\
\text { No. }\end{array}$ & $\begin{array}{l}\text { Mass } \\
(\mathrm{m} / \mathrm{z})\end{array}$ & $\begin{array}{c}\mathrm{IR} \\
\left(v, \mathrm{~cm}^{-1}\right)\end{array}$ & $\begin{array}{c}{ }^{1} \mathrm{H} \text { NMR }(\delta, \mathrm{ppm}) \\
\left(\mathrm{DMSO}-d_{6}\right)\end{array}$ \\
\hline $8 a$ & $\begin{array}{l}266\left(\mathrm{M}^{+}+1\right)(34) \\
265\left(\mathrm{M}^{+}\right)(100)\end{array}$ & $\begin{array}{l}3450(\text { brs, } 2 \mathrm{NH}) \\
17051686 \\
(2 \mathrm{C}=\mathrm{O})\end{array}$ & $\begin{array}{l}1.03\left(\mathrm{~d}, 6 \mathrm{H}, 2 \mathrm{CH}_{3}\right), 2.02(\text { Septet, } 1 \mathrm{H}, \mathrm{CH}), 2.39(\mathrm{~s}, \\
\left.3 \mathrm{H}, \mathrm{CH}_{3}\right), 2.48\left(\mathrm{~s}, 2 \mathrm{H}, \mathrm{CH}_{2}\right), 8.60(\mathrm{brs}, 2 \mathrm{H}, 2 \mathrm{NH}, \\
\left.\mathrm{D}_{2} \mathrm{O} \text { exchangeable), } 13.20 \text { (brs, } 1 \mathrm{H}, \mathrm{NH}\right)\end{array}$ \\
\hline $8 b$ & & $\begin{array}{l}3397(\text { brs, } 2 \mathrm{NH}) \\
1710(\mathrm{C}=\mathrm{O}) \\
1360(\mathrm{C}=\mathrm{S})\end{array}$ & $\begin{array}{l}1.02\left(\mathrm{~d}, 6 \mathrm{H}, 2 \mathrm{CH}_{3}\right), 2.03(\text { Septet, } 1 \mathrm{H}, \mathrm{CH}), 2.41(\mathrm{~s}, \\
\left.3 \mathrm{H}, \mathrm{CH}_{3}\right), 2.51\left(\mathrm{~s}, 2 \mathrm{H}, \mathrm{CH}_{2}\right), 8.45(\text { brs, } 2 \mathrm{H}, 2 \mathrm{NH}), \\
13.30(\mathrm{brs}, 1 \mathrm{H}, \mathrm{NH})\end{array}$ \\
\hline 9 & $288\left(\mathrm{M}^{+}\right)(100)$ & $\begin{array}{l}3450-3320 \\
\left(\mathrm{brs}, 2 \mathrm{NH}_{2}\right) \\
2218(\mathrm{CN}) \\
1705(\mathrm{C}=\mathrm{O})\end{array}$ & $\begin{array}{l}1.00\left(\mathrm{~d}, 6 \mathrm{H}, 2 \mathrm{CH}_{3}\right), 2.00(\text { Septet, } 1 \mathrm{H}, \mathrm{CH}), 2.38(\mathrm{~s}, \\
\left.3 \mathrm{H}, \mathrm{CH}_{3}\right), 2.49\left(\mathrm{~s}, 2 \mathrm{H}, \mathrm{CH}_{2}\right), 8.42\left(\text { brs, } 2 \mathrm{H}, \mathrm{NH}_{2}\right), \\
13.20\left(\mathrm{brs}, 2 \mathrm{H}, \mathrm{NH}_{2}\right)\end{array}$ \\
\hline 10 & $289\left(\mathrm{M}^{+}\right)(100)$ & $\begin{array}{l}3500(\mathrm{OH}) \\
3400\left(\mathrm{brs}, \mathrm{NH}_{2}\right) \\
2215(\mathrm{CN}) \\
1712(\mathrm{C}=\mathrm{O})\end{array}$ & $\begin{array}{l}1.01\left(\mathrm{~d}, 6 \mathrm{H}, 2 \mathrm{CH}_{3}\right), 2.04(\text { Septet, } 1 \mathrm{H}, \mathrm{CH}), 2.40(\mathrm{~s}, \\
\left.3 \mathrm{H}, \mathrm{CH}_{3}\right), 2.50\left(\mathrm{~s}, 2 \mathrm{H}, \mathrm{CH}_{2}\right), 8.23\left(\mathrm{brs}, 2 \mathrm{H}, \mathrm{NH}_{2},\right. \\
\left.\mathrm{D}_{2} \mathrm{O} \text { exchangeable), } 12.20 \text { (brs, } 1 \mathrm{H}, \mathrm{OH}\right)\end{array}$ \\
\hline $11 \mathrm{a}$ & $\begin{array}{l}279\left(\mathrm{M}^{+}+1\right)(29) \\
278\left(\mathrm{M}^{+}\right)(79)\end{array}$ & $\begin{array}{l}3390(\text { brs, NH) } \\
2924,2213(\mathrm{CN}) \\
1705(\mathrm{C}=\mathrm{O})\end{array}$ & $\begin{array}{l}1.02\left(\mathrm{~d}, 6 \mathrm{H}, 2 \mathrm{CH}_{3}\right), 1.34\left(\mathrm{t}, 3 \mathrm{H}, \mathrm{CH}_{3}\right), 2.01(\text { Septet, } \\
1 \mathrm{H}, \mathrm{CH}), 2.41\left(\mathrm{~s}, 3 \mathrm{H}, \mathrm{CH}_{3}\right), 2.52\left(\mathrm{~s}, 2 \mathrm{H}, \mathrm{CH}_{2}\right), 3.89 \\
\left(\mathrm{q}, 2 \mathrm{H}, \mathrm{CH}_{2}\right), 8.00(\mathrm{~s}, 1 \mathrm{H}, \text { methylenic proton), } \\
13.00\left(\text { brs, } 1 \mathrm{H}, \mathrm{NH}, \mathrm{D}_{2} \mathrm{O} \text { exchangeable) }\right.\end{array}$ \\
\hline $11 b$ & & $\begin{array}{l}3385(\text { brs, NH) } \\
2215(\mathrm{CN}) \\
1708(\mathrm{C}=\mathrm{O})\end{array}$ & $\begin{array}{l}1.01\left(\mathrm{~d}, 6 \mathrm{H}, 2 \mathrm{CH}_{3}\right), 1.35\left(\mathrm{t}, 3 \mathrm{H}, \mathrm{CH}_{3}\right), 2.00(\text { Septet, } \\
1 \mathrm{H}, \mathrm{CH}), 2.11\left(\mathrm{~s}, 3 \mathrm{H}, \mathrm{CH}_{3}\right), 2.43\left(\mathrm{~s}, 3 \mathrm{H}, \mathrm{CH}_{3}\right), 2.53 \\
\left(\mathrm{~s}, 2 \mathrm{H}, \mathrm{CH}_{2}\right), 3.91\left(\mathrm{q}, 2 \mathrm{H}, \mathrm{CH}_{2}\right), 13.10 \text { (brs, } 1 \mathrm{H}, \\
\mathrm{NH})\end{array}$ \\
\hline 12 & & $\begin{array}{l}3420\left(\text { brs, } \mathrm{NH}_{2}\right) \\
2216(\mathrm{CN}) \\
1708(\mathrm{C}=\mathrm{O})\end{array}$ & $\begin{array}{l}1.03\left(\mathrm{~d}, 6 \mathrm{H}, 2 \mathrm{CH}_{3}\right), 2.00(\text { Septet, } 1 \mathrm{H}, \mathrm{CH}), 2.40(\mathrm{~s}, \\
\left.3 \mathrm{H}, \mathrm{CH}_{3}\right), 2.51\left(\mathrm{~s}, 2 \mathrm{H}, \mathrm{CH}_{2}\right), 8.16(\mathrm{~s}, 1 \mathrm{H}, \text { methyl- } \\
\text { enic proton), } 11.50\left(\mathrm{brs}, 2 \mathrm{H}, \mathrm{NH}_{2}, \mathrm{D}_{2} \mathrm{O} \text { exchange- }\right. \\
\text { able) }\end{array}$ \\
\hline 13 & $249\left(\mathrm{M}^{+}\right)(100)$ & $\begin{array}{l}3410\left(\mathrm{brs}, \mathrm{NH}_{2}\right) \\
1704(\mathrm{C}=\mathrm{O})\end{array}$ & $\begin{array}{l}1.00\left(\mathrm{~d}, 6 \mathrm{H}, 2 \mathrm{CH}_{3}\right), 2.02(\text { Septet, } 1 \mathrm{H}, \mathrm{CH}), 2.39(\mathrm{~s}, \\
\left.3 \mathrm{H}, \mathrm{CH}_{3}\right), 2.50\left(\mathrm{~s}, 2 \mathrm{H}, \mathrm{CH}_{2}\right), 8.32(\mathrm{~s}, 1 \mathrm{H}, \text { pyrimi- } \\
\text { dine proton), } 11.50 \text { (brs, } 2 \mathrm{H}, \mathrm{NH}_{2}, \mathrm{D}_{2} \mathrm{O} \text { exchange- } \\
\text { able) }\end{array}$ \\
\hline 14 & $\begin{array}{l}264\left(\mathrm{M}^{+}\right)(100) \\
221\left(\mathrm{M}^{+}-43\right) \\
(43)\end{array}$ & $\begin{array}{l}3460-3350 \\
\left(\text { brs, } \mathrm{NH}+\mathrm{NH}_{2}\right) \\
1710(\mathrm{C}=\mathrm{O})\end{array}$ & $\begin{array}{l}1.04\left(\mathrm{~d}, 6 \mathrm{H}, 2 \mathrm{CH}_{3}\right), 2.03(\text { Septet, } 1 \mathrm{H}, \mathrm{CH}), 2.42(\mathrm{~s}, \\
\left.3 \mathrm{H}, \mathrm{CH}_{3}\right), 2.53\left(\mathrm{~s}, 2 \mathrm{H}, \mathrm{CH}_{2}\right), 8.28(\mathrm{~s}, 1 \mathrm{H}, \text { pyrimi- } \\
\text { dine proton), } 8.65,11.50\left(2 \mathrm{brs}, 3 \mathrm{H}\left(\mathrm{NH}, \mathrm{NH}_{2}\right) \text {, }\right. \\
\mathrm{D}_{2} \mathrm{O} \text { exchangeable) }\end{array}$ \\
\hline
\end{tabular}

$10 \%(V / V)$ Tween 80 suspension intraperitonially. One group was administered acetylsalicylic acid intraperitoneally in a dose of $200 \mathrm{mg} \mathrm{kg}^{-1}$ once daily for 3 days. The remaining groups of animals were administered test compounds intraperitoneally in a dose of $20 \mathrm{mg} \mathrm{kg}^{-1}$. On the fourth day, pylorus was ligated as per the method of Shay et al. (19); animals were fasted for $36 \mathrm{~h}$ before the pylorus ligation procedure. Four hours after the ligation, animals were sacrificed. The stomach was removed and opened along the greater curvature. Ulcer index was determined by the method of Ganguly and Bhatnagar (20) and is given in Table V. 
A.-R. B. A. El-Gazzar et al.: Synthesis and biological evaluation of thieno[2,3- $d]$ pyrimidine derivatives for anti-inflammatory, analgesic and ulcerogenic activity, Acta Pharm. 57 (2007) 395-411.

Table III. Percent anti-inflammatory activity of test compounds ${ }^{a, b, c}$

\begin{tabular}{|c|c|c|c|c|c|c|c|c|}
\hline \multirow[b]{2}{*}{ Compd. } & \multicolumn{2}{|c|}{1 hour } & \multicolumn{2}{|c|}{2 hours } & \multicolumn{2}{|c|}{3 hours } & \multicolumn{2}{|c|}{4 hours } \\
\hline & $\begin{array}{c}\text { Edema rate } \\
(\%)\end{array}$ & $\begin{array}{l}\text { Pot. } \\
(\%)\end{array}$ & $\begin{array}{c}\text { Edema rate } \\
(\%)\end{array}$ & $\begin{array}{l}\text { Pot. } \\
(\%)\end{array}$ & $\begin{array}{c}\text { Edema rate } \\
(\%)\end{array}$ & $\begin{array}{l}\text { Pot. } \\
(\%)\end{array}$ & $\begin{array}{l}\text { Edema rate } \\
(\%)\end{array}$ & $\begin{array}{l}\text { Pot. } \\
(\%)\end{array}$ \\
\hline Control & $34.8 \pm 3.1^{\mathrm{f}}$ & 0 & $53.0 \pm 1.9^{f}$ & 0 & $82.0 \pm 3.3^{f}$ & 0 & $91.6 \pm 4.5^{f}$ & 0 \\
\hline $4 a$ & $\begin{array}{c}24.2 \pm 0.9^{\mathrm{e}, \mathrm{f}} \\
\quad(30.5)\end{array}$ & 54 & $\begin{array}{c}42.0 \pm 2.0^{f} \\
(20.8)\end{array}$ & 36 & $\begin{array}{c}64.0 \pm 1.9^{\mathrm{e}, \mathrm{f}} \\
(21.9)\end{array}$ & 51 & $\begin{array}{c}74.6 \pm 3.3^{\mathrm{e}, \mathrm{f}} \\
(18.6)\end{array}$ & 40 \\
\hline $4 b$ & $\begin{array}{c}24.2 \pm 0.9^{\mathrm{e}, \mathrm{f}} \\
(30.5)\end{array}$ & 54 & $\begin{array}{c}42.0 \pm 2.0^{f} \\
(20.8)\end{array}$ & 36 & $\begin{array}{c}64.0 \pm 1.9^{\mathrm{e}, \mathrm{f}} \\
(21.9)\end{array}$ & 51 & $\begin{array}{c}74.5 \pm 3.3^{\mathrm{e}, \mathrm{f}} \\
(18.6)\end{array}$ & 40 \\
\hline $4 c$ & $\begin{array}{c}31.2 \pm 2.5^{f} \\
(10.1)\end{array}$ & 18 & $\begin{array}{c}52.3 \pm 4.8^{f} \\
(1.2)\end{array}$ & 2 & $\begin{array}{c}79.5 \pm 2.3^{f} \\
(2.8)\end{array}$ & 7 & $\begin{array}{c}82.9 \pm 1.9^{f} \\
(9.5)\end{array}$ & 20 \\
\hline $4 d$ & $\begin{array}{c}31.2 \pm 2.5^{f} \\
(10.1)\end{array}$ & 18 & $\begin{array}{c}52.3 \pm 4.8^{f} \\
(1.2)\end{array}$ & 2 & $\begin{array}{c}79.5 \pm 2.3^{\mathrm{f}} \\
(2.8)\end{array}$ & 7 & $\begin{array}{c}82.9 \pm 1.9^{f} \\
(9.5)\end{array}$ & 20 \\
\hline $4 e$ & $\begin{array}{c}24.2 \pm 0.9^{\mathrm{e}, \mathrm{f}} \\
(30.5)\end{array}$ & 54 & $\begin{array}{c}42.0 \pm 2.0^{f} \\
(20.8)\end{array}$ & 36 & $\begin{array}{c}64.0 \pm 1.9^{\mathrm{e}, \mathrm{f}} \\
(21.8)\end{array}$ & 51 & $\begin{array}{c}74.5 \pm 3.3^{\mathrm{e}, \mathrm{f}} \\
(18.6)\end{array}$ & 40 \\
\hline $4 f$ & $\begin{array}{c}31.2 \pm 2.5^{f} \\
(10.1)\end{array}$ & 18 & $\begin{array}{c}52.3 \pm 4.8^{f} \\
(1.2)\end{array}$ & 2 & $\begin{array}{c}79.5 \pm 2.3^{f} \\
(2.8)\end{array}$ & 7 & $\begin{array}{c}82.9 \pm 1.9^{f} \\
(9.5)\end{array}$ & 20 \\
\hline $5 a$ & $\begin{array}{c}31.1 \pm 1.8^{f} \\
(10.5)\end{array}$ & 15 & $\begin{array}{c}52.5 \pm 1.9^{f} \\
(0.9)\end{array}$ & 2 & $\begin{array}{c}81.7 \pm 4.1^{\mathrm{f}} \\
(0.1)\end{array}$ & 0 & $\begin{array}{c}90.9 \pm 3.0 \\
(0.8)\end{array}$ & 2 \\
\hline $5 b$ & $\begin{array}{c}24.2 \pm 0.9^{\mathrm{e}, \mathrm{f}} \\
(30.5)\end{array}$ & 54 & $\begin{array}{c}41.9 \pm 2.0^{f} \\
(20.8)\end{array}$ & 36 & $\begin{array}{c}64.0 \pm 1.9^{\mathrm{e}, \mathrm{f}} \\
(21.8)\end{array}$ & 51 & $\begin{array}{c}74.5 \pm 3.3^{\mathrm{e}, \mathrm{f}} \\
(18.6)\end{array}$ & 40 \\
\hline 6 & $\begin{array}{c}25.7 \pm 1.2^{f} \\
(25.9)\end{array}$ & 46 & $\begin{array}{c}40.9 \pm 3.1^{\mathrm{e}, \mathrm{f}} \\
(22.8)\end{array}$ & 39 & $\begin{array}{c}62.3 \pm 4.2^{\mathrm{e}, \mathrm{f}} \\
(23.9)\end{array}$ & 56 & $\begin{array}{c}71.6 \pm 3.3^{\mathrm{e}, \mathrm{f}} \\
(21.8)\end{array}$ & 47 \\
\hline 7 & $\begin{array}{c}25.7 \pm 1.2^{\mathrm{e}, \mathrm{f}} \\
(25.9)\end{array}$ & 46 & $\begin{array}{c}40.9 \pm 3.1^{\mathrm{f}} \\
(1.2)\end{array}$ & 39 & $\begin{array}{c}62.3 \pm 4.2^{\mathrm{e}, \mathrm{f}} \\
(23.9)\end{array}$ & 56 & $\begin{array}{c}71.6 \pm 3.3^{\mathrm{e}, \mathrm{f}} \\
(21.8)\end{array}$ & 47 \\
\hline $8 a$ & $\begin{array}{c}31.2 \pm 2.5^{f} \\
(10.1)\end{array}$ & 18 & $52.3 \pm 4.8^{f}$ & 2 & $\begin{array}{c}79.5 \pm 2.3^{f} \\
(2.8)\end{array}$ & 7 & $\begin{array}{c}82.9 \pm 1.9^{f} \\
(9.5)\end{array}$ & 21 \\
\hline $8 b$ & $\begin{array}{c}31.1 \pm 1.8^{f} \\
(10.5)\end{array}$ & 15 & $\begin{array}{c}52.5 \pm 1.9^{f} \\
(0.9)\end{array}$ & 2 & $\begin{array}{c}81.7^{ \pm} \pm 4.1^{\mathrm{f}} \\
(0.1)\end{array}$ & 0 & $\begin{array}{c}90.9 \pm 3.0 \\
(0.8)\end{array}$ & 2 \\
\hline $\begin{array}{l}\text { Indo- } \\
\text { methacin }\end{array}$ & $\begin{array}{c}15.0 \pm 0.9^{\mathrm{e}} \\
(56.9)\end{array}$ & 100 & $\begin{array}{c}22.2 \pm 0.8^{\mathrm{e}} \\
(58.2)\end{array}$ & 100 & $\begin{array}{c}47.0 \pm 5.0^{\mathrm{e}} \\
(42.6)\end{array}$ & 100 & $\begin{array}{c}49.1 \pm 2.7^{\mathrm{e}} \\
(46.4)\end{array}$ & 100 \\
\hline
\end{tabular}

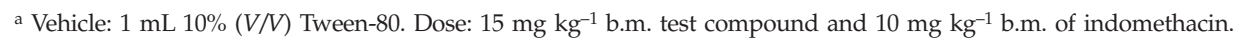

b Values represent the mean \pm SEM $(n=6)$.

c Each value in parentheses indicates the percentage inhibition rate.

d The potency (pot.) was calculated compared to the reference drug indomethacin.

e Significantly different from control (Dunnett's test): $p<0.05$.

f Significantly different from indomethacin (Dunnett's test): $p<0.05$.

\section{RESULTS AND DISCUSSION}

According to Gewald et al. (11), heating under stirring of a mixture of ethylcyanoacetate, sulfur, benzylmethyl ketone and diethylamine in absolute ethanol for 8 hours afforded 2-amino-4-methyl-5-phenyl-thiophene-3-ethylcarboxylate $\mathbf{1}$ (85\%). The latter compound was cyclized by refluxing in a mixture of potassium thiocyanate and dioxane in 
A.-R. B. A. El-Gazzar et al.: Synthesis and biological evaluation of thieno[2,3-d]pyrimidine derivatives for anti-inflammatory, analgesic and ulcerogenic activity, Acta Pharm. 57 (2007) 395-411.

Table IV. Peripheral analgesic activity ${ }^{a}$

\begin{tabular}{ccc}
\hline Compd. & No. of writhes in 20 min & Protection $(\%)$ \\
\hline Control & $48.0 \pm 2.2^{\mathrm{d}}$ & 0 \\
$\mathbf{4 a}$ & $23.2 \pm 1.4^{\mathrm{c}}$ & 52 \\
$\mathbf{4 b}$ & $31.0 \pm 1.6^{\mathrm{c}, \mathrm{d}}$ & 35 \\
$\mathbf{4} \mathbf{c}$ & $30.2 \pm 1.7^{\mathrm{c}, \mathrm{a}}$ & 37 \\
$\mathbf{4} \mathbf{d}$ & $31.0 \pm 1.6^{\mathrm{c}, \mathrm{d}}$ & 35 \\
$\mathbf{4}$ & $41.3 \pm 2.4^{\mathrm{d}}$ & 14 \\
$\mathbf{4} \mathbf{f}$ & $45.2 \pm 1.8^{\mathrm{d}}$ & 6 \\
$\mathbf{5 a}$ & $31.3 \pm 2.5^{\mathrm{c}, \mathrm{d}}$ & 35 \\
$\mathbf{5 b}$ & $19.6 \pm 0.5^{\mathrm{c}}$ & 59 \\
$\mathbf{6}$ & $19.0 \pm 1.2^{\mathrm{c}}$ & 60 \\
$\mathbf{7}$ & $19.6 \pm 0.5^{\mathrm{c}}$ & 59 \\
$\mathbf{8 a}$ & $45.8 \pm 2.9^{\mathrm{d}}$ & 5 \\
$\mathbf{8 b}$ & $45.8 \pm 2.9^{\mathrm{d}}$ & 5 \\
\hline Acetylsalicylic acid & $22.6 \pm 2.0^{\mathrm{c}}$ & 53 \\
\hline
\end{tabular}

a Vehicle: $1 \mathrm{~mL}$ of distilled water. Dose: $15 \mathrm{mg} \mathrm{kg}^{-1}$ b.m. of test compound and $100 \mathrm{mg} \mathrm{kg}^{-1} \mathrm{~b} . \mathrm{m}$. of acetylsalicylic acid.

$\mathrm{b}$ Values represent the mean $\pm \operatorname{SEM}(n=6)$.

c Significantly different from control (Dunnett's test): $p<0.05$.

d Significantly different from acetylsalicylic (Dunnett's test): $p<0.05$.

Table V. Ulcerogenicity index

\begin{tabular}{cc}
\hline Compd. & Ulcer idex \\
\hline Control & $0.9 \pm 0.1^{\mathrm{c}}$ \\
$\mathbf{4 a}$ & $1.1 \pm 0.3^{\mathrm{d}}$ \\
$\mathbf{4 b}$ & $0.9 \pm 0.3^{\mathrm{c}}$ \\
$\mathbf{4} \mathbf{c}$ & $1.2 \pm 0.3^{\mathrm{d}}$ \\
$\mathbf{4 d}$ & $0.7 \pm 0.2^{\mathrm{c}}$ \\
$\mathbf{4} \mathbf{e}$ & $1.1 \pm 0.2^{\mathrm{d}}$ \\
$\mathbf{4 f}$ & $0.8 \pm 0.2^{\mathrm{c}}$ \\
$\mathbf{5 a}$ & $0.9 \pm 0.2^{\mathrm{c}}$ \\
$\mathbf{5 b}$ & $1.1 \pm 0.2^{\mathrm{d}}$ \\
$\mathbf{6}$ & $1.1 \pm 0.2^{\mathrm{d}}$ \\
$\mathbf{7}$ & $0.9 \pm 0.3^{\mathrm{c}}$ \\
$\mathbf{8 a}$ & $0.9 \pm 0.2^{\mathrm{c}}$ \\
$\mathbf{8 b}$ & $1.1 \pm 0.3^{\mathrm{d}}$ \\
Acetylsalicylic acid & $1.7 \pm 0.4^{\mathrm{d}}$ \\
\hline
\end{tabular}

a Vehicle: $1 \mathrm{~mL}$ of $10 \%(V / V)$ of Tween-80. Dose: $20 \mathrm{mg} \mathrm{kg}^{-1}$ b.m. of test compound and $200 \mathrm{mg} \mathrm{kg}^{-1}$ b.m. of acetylsalicylic acid.

$\mathrm{b}$ Each value represents the mean $\pm \operatorname{SEM}(n=6)$.

Significantly different from acetylsalicylic acid: ${ }^{\mathrm{c}} p<0.05,{ }^{\mathrm{d}} p<0.01$. 
<smiles></smiles><smiles>CC(=O)CC(=O)CC(C)C</smiles><smiles>CC(=O)Cc1c(C(C)C)sc(N)c1C#N</smiles>

Scheme 1

the presence of concentrated hydrochloric acid affording 5-methyl-6-phenyl-2-thioxo-thieno[2,3-d]pyrimidine derivative 2 (Scheme 1). The ${ }^{1} \mathrm{H}$ NMR spectrum of the resulting product 2 is in agreement with the given structure and literature (21). Stirring a mixture of malononitrile, 6-methyl-heptane-2,4-dione, sulfur and diethylamine in absolute ethanol afforded acetone-1-(2-amino-5-isopropyl-thiophene-3-carbonitrile derivative 3 (73\%) (Scheme 1). Also, the latter compound was used as a good source to enrich the synthesis of heterocyclic chemistry with several new thieno-pyrimidines, thienothiazine and thienopyridine derivatives.

As part of our continuing program on the synthesis of various functionalized mono-heterocyclic and annelated heterocyclic derivatives $(22,23)$, we report here a new synthetic strategy for the preparation of functionalized thieno[2,3- $d][1,2,4]-5 \mathrm{H}-1,3-$ disubstituted-triazolo[4,3-a]pyrimidine-5-one derivatives $4 \mathbf{a}-\mathbf{f}$ via reactions of hydrazonoyl chlorides with 5-methyl-6-phenyl-2-thioxo-thieno[2,3-d]pyrimidine (2) (Scheme 2). Beside the correct values of elemental analysis, the IR, ${ }^{1} \mathrm{H}$ NMR and mass spectra of compounds $\mathbf{4 a}-\mathbf{f}$ are in agreement with the assigned structures. The N-3 nitrogen atom and not the $\mathrm{N}-1$ nitrogen atom was involved in the cyclization to form adduct 4 and not isomer $4^{\prime}$.

2-Amino-5-isopropyl-4-(2-oxo-propyl)-thiophene-3-carbonitrile (3) behaved as a typical $\beta$-enaminonitrile derivative due to the presence of amino and carbonitrile groups at positions 2 and 3, respectively, attached to the thiophene ring, which is considered as a precursor in the synthesis of various heterocyclic derivatives with several new azolothienopyrimidines, thienothiazine and thienopyrimidines (24). Therefore, heating under reflux of compound 3 with aliphatic acids, namely, formic and acetic acids for 6 hours 


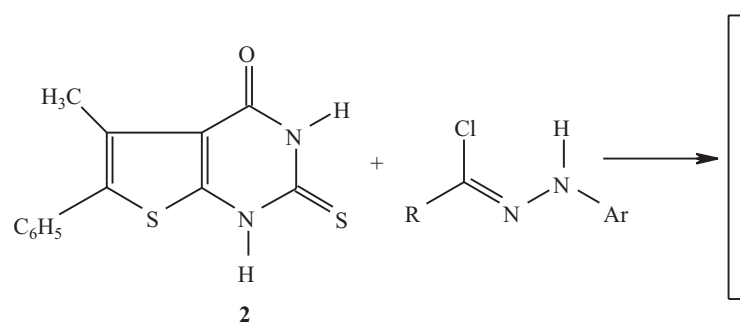

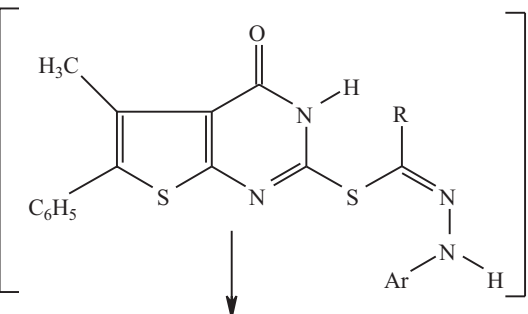<smiles>[Y][CH]c1sc2nc3n(c(=O)c2c1C)C([R])(S)N([Al])N3[CH]C</smiles><smiles>[R]C1=NN([AlH2])C2(Nc3sc(CCCCCC)c(C)c3C(=O)N2[2H])S1</smiles>

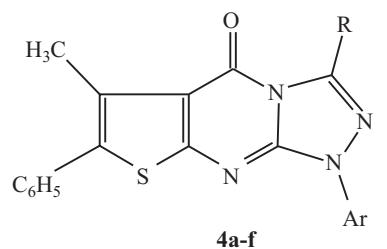

4a-f<smiles>[R]c1nn([AlH2])c2nc(=O)c3c(C)c(CCCC)sc3n12</smiles><smiles></smiles>

a: $\mathrm{R}=\mathrm{Ar}=\mathrm{C}_{6} \mathrm{H}_{5}$
b: $\mathrm{R}=\mathrm{COCH}_{3}, \mathrm{Ar}=p-\mathrm{C}_{6} \mathrm{H}_{4}-\mathrm{Cl}$
c: $\mathrm{R}=\mathrm{COCH}_{3}, \mathrm{Ar}=p-\mathrm{C}_{6} \mathrm{H}_{4}-\mathrm{OCH}_{3}$

d: $\mathrm{R}=\mathrm{COCH}_{3}, \mathrm{Ar}=p-\mathrm{C}_{6} \mathrm{H}_{4}-\mathrm{NO}_{2}$

e: $\mathrm{R}=\mathrm{COOC}_{2} \mathrm{H}_{5}, \mathrm{Ar}=\mathrm{C}_{6} \mathrm{H}_{5}$

f: $\mathrm{R}=\mathrm{COOC}_{2} \mathrm{H}_{5}, \mathrm{Ar}=p-\mathrm{C}_{6} \mathrm{H}_{4}-\mathrm{CH}_{3}$

Scheme 2

yielded thieno[2,3- $d]$-pyrimidin-4-one $(5 \mathbf{a}, \mathbf{b})$ (Scheme 3). Besides, the correct values of elemental analyses, the ${ }^{1} \mathrm{H}$ NMR spectrum, IR and mass spectra of the new compounds were in agreement with the assigned structures.

Similarly, compound 3 reacted with carbon disulphide in the presence of potassium hydroxide in absolute ethanol to yield 1-(6-isopropyl-2,4-dithioxo-1,2,3,4-tetrahydro-thieno[2,3-d]pyrimidin-5-yl)-propan-2-one (6). IR spectrum of 6 displayed absorption bands at 1350 and $1365 \mathrm{~cm}^{-1}$ of two $\mathrm{C}=\mathrm{S}$ groups. On the other hand, stirring under reflux of com- 
<smiles>[R]c1nc2sc(C(C)C)c(C(C)C)c2c(=O)[nH]1</smiles><smiles>CC(=O)c1c(C(C)C)sc2nc(N)c(C)c(N)c12</smiles>

9<smiles>CC(=O)Cc1c(C(C)C)sc2nc(O)c(C#N)c(N)c12</smiles>

10<smiles>[R]C(=O)Nc1sc(C(C)C)c(C(C)=O)c1C#N</smiles>
a: $\mathrm{R}=\mathrm{H} \quad$ b: $\mathrm{R}=\mathrm{Me}$<smiles>CC(=O)c1c(C(C)C)sc(N=CN)c1C#N</smiles>

12<smiles>CC(=O)c1c(C(C)C)sc2[nH]c(=S)[nH]c(=S)c12</smiles><smiles>CC(=O)c1c(C(C)C)sc2[nH]c(=S)sc(=N)c12</smiles><smiles></smiles><smiles>CC(=O)Cc1c(C(C)C)sc2ncn(N)c(=N)c12</smiles>

14

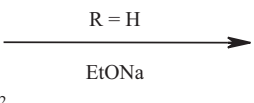

pound 3 with carbon disulphide in dry pyridine afforded the 1-(4-imino-6-isopropyl2-thioxo-1,4-dihydro-2H-thieno[2,3- $d][1,3]$ thiazin-5-yl)-propan-2-one (7). Also, when heated with urea or thiourea at $180{ }^{\circ} \mathrm{C}$, compound 3 gave 4-amino-6-isopropyl-5-(2-oxo-propyl)- $1 H$-thieno[2,3- $d$ ] pyrimidin-(2-one or 2-thione) derivatives $\mathbf{8} \mathbf{a}, \mathbf{b}$, respectively (Scheme 3 ).

When compound 3 was reacted with active methylenes, malononitrile in glacial acetic acid and ethylcyanoacetate in dry dioxane in the presence of catalytic amount of tri- 
ethylamine, it yielded the corresponding 4,6-diamino-2-isopropyl-3-(2-oxo-propyl)-thieno-[2,3-b]pyridine-5-carbonitrile (9) and 4-amino-6-hydroxy-2-isopropyl-3-(2-oxo-propyl)-thieno[2,3-b]pyridine-5-carbonitrile (10), respectively (Scheme 3). Structures 9 and 10 were tentatively preferred for this product based on its ${ }^{1} \mathrm{H}$ NMR spectrum and the IR spectra, which revealed the presence of the cyano-group around $2212 \mathrm{~cm}^{-1}$ and $\mathrm{NH}_{2}$ group around $3500 \mathrm{~cm}^{-1}$. Condensation of 3 with triethyl orthoformate or triethylorthoacetate in boiling acetic anhydride yielded the corresponding $\mathrm{N}-[3-\mathrm{cyano}-5$-isopropyl-4-(2-oxo-propyl)-thiophen-2-yl]-formamidic acid ethyl ester (11a) and N-[3-cyano-5-isopropyl-4-(2-oxo-propyl)-thiophen-2-yl]-acetimidic acid ethyl ester (11b) respectivly, with compatible IR and ${ }^{1} \mathrm{H}$ NMR data. Compound 11a reacted with ammonium hydroxide solution in absolute ethanol to give N-[3-cyano-5-isopropyl-4-(2-oxo-propyl)-thiophen-2-yl]-formamidine (12), which on boiling in absolute ethanolic sodium ethoxide solution underwent cyclization to give 1-(4-amino-6-isopropyl-thieno[2,3-d]-pyrimidin-5-yl)-propan-2-one (13) (Scheme 3). The IR spectrum of 12 displayed an absorption band at $2216 \mathrm{~cm}^{-1}(\mathrm{CN})$, while, that of 13 revealed the absence of $\mathrm{CN}$ group. Finally, stirring compound 11a with hydrazine in absolute ethanol at room temperature gave 1-(3-amino-4-imino-6-isopropyl-3,4-dihydro- thieno[2,3-d]pyrimidin-5-yl)-propan-2-one (14).

The anti-inflammatory activity data (Table III) indicated that all the test compounds protected rats from carrageenean induced inflammation. Compounds $4 \mathbf{a}, 4 \mathbf{b}, \mathbf{4 e}, 5 \mathbf{b}, \mathbf{6}$ and 7 protected rats by 36 to $56 \%$ from inflammation while the other tested compounds showed lower anti-inflamatory activity in comparison to indomethacin. This means that the fused triazolo[4,3-a]pyrimidine, pyrimidine-2,4-dithione and thieno[2,3- $d][1,3]$ thiazine possess good anti-inflammatory activity.

The results of analgesic activity indicate that all the test compounds exhibited high activity (Table IV). Triazolo[4,3-a]pyrimidine 4a with triphenyl substitution showed high activity comparable to that of acetylsalicylic acid. The compounds with acetyl or ethylester group substitution $(\mathbf{4} \mathbf{b}-\mathbf{d})$, showed moderate activity. Also, thieno[2,3- $d]$ pyrimidines $5 \mathbf{b}$ and $\mathbf{6}$ and thieno[2,3- $d][1,3]$ thiazin 7 were found to be the most active analgesic agents, even more potent than acetylsalicylic acid.

The ulcer index of the test compounds (Table V) reveals that all compounds showed a mild ulcer index as compared to acetylsalicylic acid.

\section{CONCLUSION}

New compounds, substituted thieno[2,3- $d][1,2,4]$-triazolo[4,3-a]pyrimidin-5-ones 4a, 4b , 4e 6-isopropyl-2-methyl-5-(2-oxo-propyl)-3H-thieno[2,3-d] pyrimidin-4-one (5b), 1-(6-isopropyl-2,4-dithioxo-1,2,3,4-tetrahydro-thieno[2,3-d] pyrimidin-5-yl)propan-2-one (6) and 1-(4-imino-6-isopropil-2-thioxo-1,4-dihydro-2H-thieno[2,3-d][1,3]thiazin-5-yl)propan-2-one (7) showed moderate anti-inflammatory activity. However, thieno[2,3- $d][1,2,4]-$ triazolo[4,3-a]pyrimidin-5-ones $4 \mathbf{a}-\mathbf{d}$ and 6-isopropyl-5-(2-oxo-propyl)-3H-thieno[2,3- $d$ ] pyrimidin-4-one (5a) and compounds $5 b, 6$ and 7 showed good to excellent analgesic activity. 


\section{REFERENCES}

1. D. J. Brown, Pyrimidines and Their Benzo Derivatives, in Comprehensive Heterocyclic Chemistry (Ed. A. R. Katritzky and C. W. Rees), Vol. 3, Pergamon Press, Oxford 1984, p. 443.

2. B. Roth and C. Cheng, Diaminopyrimidines, in Progress in Medicinal Chemistry (Eds. G. B. Ellis and G. E. West), Vol. 19, Elsevier Biomedical Press, New York 1982, p. 267.

3. M. S. A. E.-A. El-Gaby, S. G. Abdel-Hamide, M. M. Ghorab and S. M. El-Sayed, Synthesis and anticancer activity in vitro of some new pyrimidines, Acta Pharm. 49 (1999) 149-158.

4. C. R. Petrie, H. B. Cottam, P. A. Mckernan, R. K. Robins and G. R. Revankar, Synthesis and biological activity of 6-azacadeguomycin and certain 2,4,6-trisubstituted pyrazolo[3,4- $d$ ]-pyrimidine ribonucleosides, J. Med. Chem. 28 (1985) 1010-1016.

5. M. N. Nasr and M. M. Gineinah, Pyrido[2,3- $d]$ pyrimidines and pyrimido $\left[5^{\prime}, 4^{\prime}: 5,6\right]-$ pyrido $[2,3-d]$ pyrimidines as new antiviral agents: Synthesis and biological activity, Arch. Pharm. 335 (2002) 289-295; DOI: 10.1002/1521-4184(200208)335:6<289.

6. P. G. Baraldi, M. G. Pavani, M. Nunez, P. Brigidi, B. Vitali, R. Gambari and R. Romagnoli, Antimicrobial and antitumor activity of N-heteroimine-1,2,3-diathiazoles and their transformation in triazolo-, imidazo- and pyrazolopyrimidines, Bioorg. Med. Chem. 10 (2002) 449-456; DOI: 10. 1016/S0968-0896(01)00294-2.

7. S. M. Sondhi, M. Johar, S. Rajvanshi, S. G. Dastidar, R. Shukla, R. Raghubir and J. W. Lown, Anticancer, anti-inflammatory and analgesic activity evaluation of heterocyclic compounds synthesized by the reaction of 4-isothiocyanato-4-methylpentan-2-one with substituted $o$-phenylenediamines, $o$-diaminopyridine and (un)substituted $o$-diamino-pyrimidines, Australian J. Chem. 54 (2001) 69-74; DOI: 10.1071/CH00141.

8. A. Z. M. S. Chowdhury, M. M. Matin and M. N. Anwar, Synthesis and antimicrobial activities of fused pyrimidines: Benzothieno[2,3- $d$ ]imidazol[1,2-c]pyrimidine, Chittagong Univ. Stud. Part II 21 (1997) 79-83; ref Chem. Abstr. 130 (1999) 237530p.

9. A. M. Abdel-Fattah, A. S. Aly, F. A. Gad, M. E. A. Zaki and A. B. A. El-Gazzar, A facile synthesis of isooxazolo[5,4:4',5']thiazolo[3,2-a]thienopyrimidines, a new ring system, Phosphorus Sulfur 141 (1998) 263-281; DOI: 10.1080/10426509808033738.

10. A. M. Abdel-Fattah, A. S. Aly, F. A. Gad, N. A. Hassan and A. B. A. El-Gazzar, Synthesis and reaction of polynuclear heterocycles, with a new ring system, Phosphorus Sulfur 163 (2000) 1-27; DOI: $10.1080 / 10426500008046607$.

11. A. K. Gewald, E. Schinke and H. Bottcher, 2-Amino-thiophene aus methylenaktiven Nitrilen, Carbonylverbindungen und Schwefel, Chem. Ber. 99 (1966) 94-100.

12. P. Wolkoff, T. S. Nemeth and S. M. Gibson, Reaction of hydrazonyl halides with derivatives of thiourea and thiosemicarbazide; A new source of C-amino- and C-hydrazino-1,2,4-triazoles, Can. J. Chem. 53 (1975) 3211-3215.

13. A. F. Hegarty, M. Cashoman, J. B. Aylward and F. L. Scott, Ortho-group participation in azocarbonium ion and 1,3-dipolar ion formation, J. Chem. Soc. B 57 (1971) 1879-1883.

14. Guide to the Care and Use of Experimental Animals, Vol. 1, $2^{\text {nd }}$ ed. (Eds. E. D. Olfert, B. M. Cross and A. A. McWilliam), Canadian Council of Animal Care, Ottawa 1993.

15. C. A. Winter, E. A. Risley and G. W. Nuss, Carrageenin-induced edema in hind paw of the rats as an assay anti-inflammatory drugs, Proc. Soc. Exp. Biol. Med. III (1962) 544-547.

16. J. Miño, V. Moscatelli, O. Hnatyszyn, S. Gorzalczany, C. Acevedo and G. Ferraro, J. Pharmacol. Res. 50 (2004) 59-63; DOI: 10.1016/j.phrs.2003.12.016.

17. H. D. J. Collier, L. C. Dinnin, C. A. Johnson and C. Schneider., The abdominal response and its suppression by analgestic drugs in the mouse Br. J. Pharmacol. Chemother. 32 (1968) 295-310.

18. R. K. Goel, A. Chakrabarthi and A. K. Sanyal, Planta Med. 29 (1985) 85-88. 
A.-R. B. A. El-Gazzar et al.: Synthesis and biological evaluation of thieno[2,3-d]pyrimidine derivatives for anti-inflammatory, analgesic and ulcerogenic activity, Acta Pharm. 57 (2007) 395-411.

19. H. Shay, S. A. Komarav, S. E. Fels, D. Meraza, M. Gruenstein and H. Siplet, Gastroenterology 5 (1994) 43-61.

20. A. K. Ganguly and O. P. Bhatnagar, Can. J. Physiol. Pharmacol. 51 (1973) 748-750.

21. A. M. Abdel-Fattah, A. S. Aly, F. Abdel-Motti, N. A. Hassan and H. A. Aly, Synthesis and reaction of some thienopyrimidine derivatives and some of photooxidation, Egyptian J. Chem. 38 (1995) 627-633.

22. A. S. Aly, A. B. A. El-Gazzar and H. A. R. Hussien, Synthesis and reactions of polynuclear heterocycles: Azolothienopyrimidines and thienothiazolopyrimidines, Phosphorus Sulfur 181 (2006) 2771-2784; DOI: 10.1080/10426500600865293.

23. A. B. A. El-Gazzar, H. A. R. Hussien and A. S. Aly, Synthesis of some derivatives derived from 1,2,3,4-tetrahydrocyclo-hepteno[4,5]thieno[2,3-d]pyrimidine, Phosphorus Sulfur 182 (2007) 35-56; DOI: $10.1080 / 10426500600864536$.

24. B. Cacciari and G. Spalluto, Facile and versatile route to the synthesis of fused 2-pyridones: Useful intermediates for polycyclic systems, Synthetic Commun. 36 (2006) 1177-1183; DOI: 10. $1080 / 00397910500514063$.

\author{
$S A \check{Z} E T A K$
}

\title{
Sinteza i protuupalno, analgetsko i ulcerogeno djelovanje derivata tieno[2,3- $d]$ pirimidina
}

ABDEL-RAHMAN B. A. EL-GAZZAR, HODA. A. R. HUSSEIN i HEND. N. HAFEZ

Reakcijom derivata 5-metil-6-fenil-2-tioksotieno[2,3-d]pirimidona (2) s hidrazonoil kloridima dobiveni su triazolotienopirimidoni $4 \mathbf{a}-\mathbf{f}$, a reakcijom aceton-1-(2-amino-5-izopropil-tiopen-3-karbonitrila (3) s funkcionalnim i bifunkcionalnim spojevima dobiveni su produkti 5-11. Novi spojevi imaju slično protuupalno, analgetsko i ulcerogeno djelovanje kao i indometacin, odnosno acetilsalicilna kiselina.

Ključne riječi: triazolotienopirimidin, tienopirimidini, tieno[2,3-d][1,3]tiazin, tienopiridini, analgetsko, protuupalno, ulcerogeno djelovanje

Photochemistry Department (Heterocyclic Unit), National Research Center, Dokki, Giza, Egypt 